\title{
One Pot Two Step Synthesis of 2-Arylidenehydrazinyl-4-arylthiazole
}

\author{
PRAVIN TATYARAM TRYAMBAKE* \\ Department of Chemistry, S.N. Arts, D. J. M. Commerce and B. N. S. Science College, \\ Sangamner, Tal-Sangamner, Dist- Ahmednagar- 422605 (MS), India. \\ *Corresponding author E-mail: pravintryambake21@ rediffmail.com \\ http://dx.doi.org/10.13005/ojc/340360 \\ (Received: October 23, 2017; Accepted: January 19, 2018)

\begin{abstract}
An efficient, simple one pot, two step procedure has been developed for the synthesis of 2-arylidenehydrazinyl-4-arylthiazole. The reaction of aromatic aldehyde, thiosemicarbazide and phenacyl bromide gave the desired products in good yield. The first reaction product thiosemicarbazone was obtained on reaction with aromatic aldehyde and thiosemicarbazide; without isolating this directly treated with phenacyl bromide in presence of acidic buffer at room temperature desired product was obtained with simple workup procedure.
\end{abstract}

Keywords: Thiosemicarbazone, Phenacyl bromide, Thiazole, Synthesis, One pot.

\section{INTRODUCTION}

Thiazoles are nitrogen and sulphur containing heterocyclic compound. Thiazoles and their derivatives are found to be associated with various biological activities such as antifungal, anti-inflammatory activities ${ }^{1-3}$, antibacterial ${ }^{4-6}$, anti $\mathrm{HIV}^{7}$, pesticidal ${ }^{8}$, antiprotozoal ${ }^{9}$ anticancer ${ }^{10,11}$, antitumor ${ }^{12}$, hypertension ${ }^{13}$. The synthesis of thiazole derivatives is important for their wide range of pharmaceutical and biological properties. Regarding the synthesis of hydrazinyl-thiazoles, general procedure has been reported in the literature. The cyclization of thiosemicarbazones with $\alpha$-halocarbonyl compounds in alcohol under reflux resulted in hydrazinyl-thiazoles derivatives ${ }^{14}$. Now people are working on development of new methods for organic compounds by considering the minimum steps. One of the aspect of method development is one pot two step reaction ${ }^{15-18}$, which involves the isolation of final product and not the intermediate products. So this is less time consuming aspect. By keeping in mind, in this procedure, the reaction of aromatic aldehyde with thiosemicarbazide in presence of sulphamic acid as catalyst $(10 \mathrm{mmol})^{19}$ in ethanol was stirred at room temperature gave thiosemicarbazone of corresponding aldehyde. Without isolation of thiosemicarbazone the same flask charged with phenacyl bromide and acidic buffer (scheme 1). The reaction mixture was further stirred till completion of reaction. The synthesized molecules were supported by IR, ${ }^{1} \mathrm{H}-\mathrm{NMR},{ }^{13} \mathrm{C}-\mathrm{NMR}$ and mass spectral data. 


\section{MATERIAL AND METHODS}

Melting points were determined in open capillary tubes and are uncorrected. IR spectra $(\mathrm{KBr})$ were recorded on a Shimadzu IR-408 spectrometer. ${ }^{1} \mathrm{H}$ and ${ }^{13} \mathrm{C}$ NMR spectra were determined in DMSO-d6 solution on a Bruker Avance II $(400 \mathrm{MHz})$ NMR Spectrometer and TMS was used as an internal standard. Mass spectra were recorded on a Waters, Q-Tof Micromass (LC-MS).

General procedure for the synthesis of 2-Arylidenehydrazinyl-4-(4-methoxy phenyl) thiazole 2(a-j)

The mixture of thiosemicarbazide (1 mmole), aromatic aldehyde (1 mole) and sulphamic acid (10 mole \%) was taken in $10 \mathrm{ml}$ ethanol in a round bottomed flask, the mixture was stirred at room temperature for 10-20 min., the completion of reaction was monitored by TLC. Further sodium acetate $(3 \mathrm{mmol})$ and acetic acid $(0.5 \mathrm{ml})$ and 4-methoxy phenacyl bromide $(1 \mathrm{mmol})$ was added to the above reaction mixture and stirred till completion of reaction, monitored by TLC. Then reaction mixture was poured into ice cold water. Separated solid was filtered, dried and purified by recrystallization.

Reagents and Conditions: (a) Sulphamic acid (10 mol \%), EtOH, Stir at room temperature (b) 4-methoxy phenacyl bromide (1 mmole), sodium acetate $(3 \mathrm{mmol})$, glacial acetic acid $(0.5 \mathrm{ml})$, stir at room temperature.<smiles>COc1ccc(-c2csc(NC=NC(C)C)n2)cc1</smiles>

1a-h

2a-h

Scheme 1

Spectroscopic data for synthesized compounds 2-(2-(3-chlorobenzylidene)hydrazinyl)-4(4-methoxyphenyl)thiazole (2a)

$\left(\mathrm{KBr}, \operatorname{mmax} \mathrm{cm}^{-1}\right): 3300(-\mathrm{NH}), 1570(\mathrm{C}=\mathrm{N}$ azomethine), $1487(\mathrm{C}=\mathrm{N}$ thiazole $), 1442,1342$, 1251; 1141(-Cl); ${ }^{1} \mathrm{H}$ NMR (DMSO-d6, $400 \mathrm{MHz}$ ) d (ppm): 3.80 (s, 3H, $\left.-\mathrm{OCH}_{3}\right), 6.92(\mathrm{~d}, \mathrm{~J}=7.242 \mathrm{H}$, p-anisyl Ar-H), 6.90 (s, 1H, Ar-H), $7.32(\mathrm{~d}, 1 \mathrm{H}, \mathrm{Ar}-\mathrm{H})$, $7.37(\mathrm{t}, 1 \mathrm{H}, \mathrm{Ar}-\mathrm{H}), 7.54(\mathrm{~d}, 1 \mathrm{H}, \mathrm{Ar}-\mathrm{H}), 7.64(\mathrm{~s}, 1 \mathrm{H}$, thiazole $\mathrm{H}), 7.76$ (d, J=8.68, 2H, p-anisyl Ar-H), 7.96 (s, $1 \mathrm{H},-\mathrm{N}=\mathrm{CH}) ; 12.07$ (s, $1 \mathrm{H},-\mathrm{NH}-) ;{ }^{13} \mathrm{C}$ NMR (400 $\mathrm{MHz}, \mathrm{DMSO}-\mathrm{d} 6): \delta 54.8,101.1,113.6,124.6,125.2$, 126.6, 127.4, 128.3, 130.0, 133.7, 138.9, 136.5, 150.4, 158.6, 167.9; MS: $M+1=344.1$

2-(2-(2-chlorobenzylidene)hydrazinyl)-4(4-methoxyphenyl)thiazole (2b):

IR (KBr, mmax cm-1): $3064(-\mathrm{NH}), 1597$ $(\mathrm{C}=\mathrm{N}$ azomethine), $1491(\mathrm{C}=\mathrm{N}$ thiazole $), 1473$, 1365, 1246; 1139(-Cl); ${ }^{1} \mathrm{H}$ NMR (DMSO-d6, 400 $\mathrm{MHz}) \mathrm{d}(\mathrm{ppm}): 3.78\left(\mathrm{~s}, 3 \mathrm{H},-\mathrm{OCH}_{3}\right), 6.98(\mathrm{~d}, \mathrm{~J}=8.7$, $2 \mathrm{H}$, p-anisyl Ar-H), 7.17 (s, $1 \mathrm{H}$, thiazole $\mathrm{H}), 7.40$ (m, 2H, Ar-H), 7.50 (d, 1H, Ar-H), 7.80 (d,J=8.7, $2 \mathrm{H}, \mathrm{p}$-anisyl Ar-H),7.94 (d, 1H, Ar-H), 8.39 (s, 1H, $-\mathrm{N}=\mathrm{CH}) ; 12.40$ (s, $1 \mathrm{H},-\mathrm{NH}-) ;{ }^{13} \mathrm{C}$ NMR $(400 \mathrm{MHz}$,
DMSO-d6): $\delta 54.9,101.4,113.7,126.1,126.7,127.1$, 127.4, 129.6, 130.0, 131.7, 132.2, 136.6, 150.4, 158.7, 167.7; MS: M+1= 344.2.

2-(2-(4-chlorobenzylidene)hydrazinyl)-4(4-methoxyphenyl)thiazole ( 2c):

IR $\left(\mathrm{KBr}, \operatorname{mmax} \mathrm{cm}^{-1}\right): 3142(-\mathrm{NH}), 1566$ ( $\mathrm{C}=\mathrm{N}$ azomethine), 1491 ( $\mathrm{C}=\mathrm{N}$ thiazole), 1435, 1361, 1249; 1134(-Cl); ${ }^{1} \mathrm{H}$ NMR (DMSO-d6, $400 \mathrm{MHz}$ ) d (ppm): 3.78(s, 3H, -OCH3), 6.98 (d, J=2.8, 2H, p-anisyl Ar-H), $7.15\left(\mathrm{~s},{ }^{1} \mathrm{H}\right.$, thiazole $\left.\mathrm{H}\right), 7.49(\mathrm{~m}, 2 \mathrm{H}$, Ar-H), 7.68 (d, 2H, Ar-H), 7.79 (d,J=2.8, 2H, p-anisyl Ar-H), $8.02(\mathrm{~s}, 1 \mathrm{H},-\mathrm{N}=\mathrm{CH}) ; 12.23(\mathrm{~s}, 1 \mathrm{H},-\mathrm{NH}-) ;{ }^{13} \mathrm{C}$ NMR (400 MHz, DMSO-d6): $\delta$ 55.0, 101.6, 113.9, 126.8, 127.4, 127.7, 128.8, 133.3, 133.5, 139.7, 150.3, 158.7, 167.9; MS: $M+1=344.2$.

4-(4-methoxyphenyl)-2-(2-(2-nitrobenzylidene) hydrazinyl)thiazole (2d)

IR (KBr, mmax cm-1): 3319 (-NH), 1577, 1521 ( $\mathrm{C}=\mathrm{N}$ azomethine), $1492(\mathrm{C}=\mathrm{N}$ thiazole), 1437 , 1340, 1249; ${ }^{1} \mathrm{H}$ NMR (DMSO-d6, $400 \mathrm{MHz}$ ) d (ppm): 3.79(s, 3H, -OCH3), 6.97 (d,J=8.7, 2H, p-anisyl Ar-H), 7.12 (s, $1 \mathrm{H}$, thiazole $\mathrm{H}), 7.67(\mathrm{t}, 1 \mathrm{H}, \mathrm{Ar}-\mathrm{H})$, 
$7.79(\mathrm{~d}, \mathrm{~J}=8.7,2 \mathrm{H}, \mathrm{p}$-anisyl Ar-H), $8.07(\mathrm{~d}, 1 \mathrm{H}$, Ar-H), $8.16(\mathrm{~s}, 1 \mathrm{H},-\mathrm{N}=\mathrm{CH}) ; 8.19(\mathrm{~d}, 1 \mathrm{H}, \mathrm{Ar}-\mathrm{H}), 8.45$ (s, $1 \mathrm{H}, \mathrm{Ar}-\mathrm{H}), 12.38$ (s, $1 \mathrm{H},-\mathrm{NH}-) ;{ }^{13} \mathrm{C}$ NMR (400 MHz, DMSO-d6): $\delta 54.9,101.8,113.9,124.2,124.4$, $126.7,128.4,128.8,133.0,135.6,147.2,158.7$, 167.6, 178.5; MS: M+1= 355.29.

\section{4-(4-methoxyphenyl)-2-(2-(3-nitrobenzylidene)} hydrazinyl)thiazole (2e)

IR $\left(\mathrm{KBr}, \operatorname{mmax} \mathrm{cm}^{-1}\right): 3064(-\mathrm{NH}), 1583$, 1527( $\mathrm{C}=\mathrm{N}$ azomethine), 1492 ( $\mathrm{C}=\mathrm{N}$ thiazole), 1435, 1348, 1249; $1 \mathrm{H}$ NMR (DMSO-d6, 400 MHz) d (ppm): 3.79(s, 3H, $\left.-\mathrm{OCH}_{3}\right), 6.97$ (d,J=8.7, 2H, p-anisyl Ar-H), $7.14(\mathrm{~s}, 1 \mathrm{H}$, thiazole $\mathrm{H}), 7.57(\mathrm{t}, 1 \mathrm{H}, \mathrm{Ar}-\mathrm{H})$, 7.72 (s, 1H, Ar-H), 8.07 (q, 2H, Ar-H), 7.78 (d,J=8.7, $2 \mathrm{H}$, p-anisyl Ar-H),7.94 (d, 1H, Ar-H), 8.44 (s, 1H, -N=CH); 12.49 (s, $1 \mathrm{H},-\mathrm{NH}-) ;{ }^{13} \mathrm{C}$ NMR (400 MHz, DMSO-d6): $\delta 54.9,101.5,113.7,120.1,122.9,126.7$, 127.4, 129.9, 131.9, 136.4, 138.2, 148.16, 158.7 , 167.7; $M S: M+1=355.29$.

\section{4-(4-methoxyphenyl)-2-(2-(4-nitrobenzylidene) hydrazinyl)thiazole (2f)}

IR $\left(\mathrm{KBr}, \operatorname{mmax} \mathrm{cm}^{-1}\right): 3313(-\mathrm{NH}), 1556$, 1527( $\mathrm{C}=\mathrm{N}$ azomethine), $1489(\mathrm{C}=\mathrm{N}$ thiazole), 1458, 1334, 1240; ' $\mathrm{H}$ NMR (DMSO-d6, $400 \mathrm{MHz}$ ) d (ppm): 3.79(s, 3H, $\left.-\mathrm{OCH}_{3}\right), 6.96(\mathrm{~d}, \mathrm{~J}=7.12,2 \mathrm{H}$, p-anisyl Ar-H), $7.14(\mathrm{~s}, 1 \mathrm{H}$, thiazole $\mathrm{H}), 7.77$ (d, $2 \mathrm{H}, \mathrm{J}=6.96, \mathrm{Ar}-\mathrm{H}), 7.89$ (d, 2H, J=8.88, Ar-H), 8.20 (s, $1 \mathrm{H},-\mathrm{N}=\mathrm{CH}) ; 8.26$ (d, 2H, J=8.84, Ar-H), 12.43 (s, $1 \mathrm{H},-\mathrm{NH}-)$ : MS: $\mathrm{M}+1=355.29$.

\section{3-((2-(4-(4-methoxyphenyl)thiazol-2-yl)hydrazono) methyl)phenol ( $2 \mathrm{~g}$ )}

${ }^{1} \mathrm{H}$ NMR (DMSO-d6, $\left.400 \mathrm{MHz}\right) \mathrm{d}(\mathrm{ppm})$ : 3.55(s, 3H, $\left.-\mathrm{OCH}_{3}\right), 6.79$ (s, $\left.1 \mathrm{H}, \mathrm{Ar}-\mathrm{H}\right), 6.96$ (d, J=8.8, 2H, p-anisyl Ar-H), 7.04 (s, 1H, Ar-H), $7.08(\mathrm{~s}, 1 \mathrm{H}$, thiazole $\mathrm{H}), 7.10(\mathrm{~s}, 1 \mathrm{H}, \mathrm{Ar}-\mathrm{H})$, 7.23 (t, 1H, Ar-H), 7.78 (d,J=8.8, 2H, p-anisyl Ar-H), 7.93 (s, 1H, - $\mathrm{N}=\mathrm{CH}$ ); 9.55 (br. s, 1H, Ar-OH),
12.49 (br. s, $1 \mathrm{H},-\mathrm{NH}-$ ); MS: $\mathrm{M}+1=325$.

2-(2-(4-bromobenzylidene)hydrazinyl)-4(4-methoxyphenyl)thiazole (2h)

IR $\left(\mathrm{KBr}, \operatorname{mmax} \mathrm{cm}^{-1}\right): 3140(-\mathrm{NH}), 1564$, ( $\mathrm{C}=\mathrm{N}$ azomethine), 1491 ( $\mathrm{C}=\mathrm{N}$ thiazole), 1456, 1361, 1249; ${ }^{1} \mathrm{H}$ NMR (DMSO-d6, $400 \mathrm{MHz}$ ) d (ppm): 3.78(s, $\left.3 \mathrm{H},-\mathrm{OCH}_{3}\right), 6.98$ (d, J=8.8, 2H, p-anisyl Ar-H), 7.15 $(\mathrm{s}, 1 \mathrm{H}$, thiazole $\mathrm{H}), 7.59(\mathrm{~d}, 1 \mathrm{H}, \mathrm{J}=6.56, \mathrm{Ar}-\mathrm{H}), 7.63$ (d, J=6.48, 2H, Ar-H), 7.79 (d, J=8.8, 2H, p-anisyl Ar-H), 8.00 (s, 1H, -N=CH); 12.22 (br. s, 1H, -NH-); ${ }^{13} \mathrm{C}$ NMR $(400 \mathrm{MHz}$, DMSO-d6): $\delta 55.0,101.6,113.9$, $122.2,126.8,127.4,128.0,131.7,133.7,139.7$, 158.7, 167.9.

4-((2-(4-(4-methoxyphenyl)thiazol-2-yl) hydrazono)methyl)phenol (2i)

IR $\left(\mathrm{KBr}, \mathrm{mmax} \mathrm{cm}^{-1}\right)$ : 3329(-OH, $\left.-\mathrm{NH}\right)$, 1535, 1512( $\mathrm{C}=\mathrm{N}$ azomethine), 1492 ( $\mathrm{C}=\mathrm{N}$ thiazole), , 1323, 1247; ${ }^{1} \mathrm{H}$ NMR (DMSO-d6, $400 \mathrm{MHz}$ ) d (ppm): $3.77\left(\mathrm{~s}, 3 \mathrm{H},-\mathrm{OCH}_{3}\right), 6.80-6.83$ (d, J=8.56, $2 \mathrm{H}$, Ar-H), 6.91-6.93 (d,J=8.76, 2H, p-anisyl Ar-H), $6.98(\mathrm{~s}, 1 \mathrm{H}$, thiazole $\mathrm{H}), 7.48(\mathrm{~d}, \mathrm{~J}=8.6,2 \mathrm{H}, \mathrm{Ar}-\mathrm{H})$, 7.77 (d,J=8.72, 2H, p-anisyl Ar-H), $7.93(\mathrm{~s}, 1 \mathrm{H},-\mathrm{N}=\mathrm{CH})$; 9.75 (br. s, $1 \mathrm{H}, \mathrm{Ar}-\mathrm{OH}), 11.82$ (br. s, $1 \mathrm{H},-\mathrm{NH}-$ ); ${ }^{13} \mathrm{CNMR}$ (400 MHz, DMSO-d6): $\delta 54.9,100.6,113.6,115.5,125.5$, 126.6, 127.6, 127.7, 141.4, 150.2, 158.6, 168.3.

\section{2-(2-(4-methoxybenzylidene)hydrazinyl)-4-(4- methoxyphenyl)thiazole (2j)}

IR $\left(\mathrm{KBr}, \operatorname{mmax} \mathrm{cm}^{-1}\right): 3151(-\mathrm{NH}), 1566$, 1512( $\mathrm{C}=\mathrm{N}$ azomethine), 1492 ( $\mathrm{C}=\mathrm{N}$ thiazole), 1361, 1246; ${ }^{1} \mathrm{H}$ NMR (DMSO-d6, $400 \mathrm{MHz}$ ) d (ppm): 3.77 $\left(\mathrm{s}, 3 \mathrm{H},-\mathrm{OCH}_{3}\right), 3.79\left(\mathrm{~s}, 3 \mathrm{H},-\mathrm{OCH}_{3}\right), 6.90-6.96$ (m, 5H, Ar-H), 7.58 (d, J=8.72, 2H, p-anisyl Ar-H), $7.76(\mathrm{~d}, \mathrm{~J}=8.72,2 \mathrm{H}, \mathrm{p}$-anisyl Ar-H), 7.95 (s, 1H, thiazole $\mathrm{H}$ ), 11.86 (br. s, $1 \mathrm{H},-\mathrm{NH}-$ ); ${ }^{13} \mathrm{C}$ NMR (400 MHz, DMSO-d6): $\delta 54.9,55.1,100.9,113.7$, $114.1,126.7,127.1,127.6,140.9,150.3,158.6$, 160.1, 168.2 .

Table 1: Physical data of newly synthesized compounds 2a-i

\begin{tabular}{ccccc}
\hline Entry & $\mathrm{R}-\mathrm{CHO}$ & Yield (\%) & m.p. $\left({ }^{\circ} \mathrm{C}\right)$ & Comp. no. \\
\hline 1 & $3-\mathrm{Cl} \mathrm{C} \mathrm{H}_{4}$ & 91 & $168-170$ & $2 \mathrm{a}$ \\
2 & $2-\mathrm{Cl} \mathrm{C}_{6} \mathrm{H}_{4}$ & 90 & $164-166$ & $2 \mathrm{~b}$ \\
3 & $4-\mathrm{Cl} \mathrm{C}_{6} \mathrm{H}_{4}$ & 92 & $234-236$ & $2 \mathrm{c}$ \\
4 & $2-\mathrm{NO}_{2} \mathrm{C}_{6} \mathrm{H}_{4}$ & 88 & $174-176$ & $2 \mathrm{~d}$ \\
5 & $3-\mathrm{NO}_{2} \mathrm{C}_{6} \mathrm{H}_{4}$ & 90 & $218-220$ & $2 \mathrm{e}$ \\
6 & $4-\mathrm{NO}_{2} \mathrm{C}_{6} \mathrm{H}_{4}$ & 87 & $228-230$ & $2 \mathrm{f}$ \\
7 & $3-\mathrm{OH} \mathrm{C}_{6} \mathrm{H}_{4}$ & 92 & $216-218$ & $2 \mathrm{~g}$ \\
8 & $4-\mathrm{Br} \mathrm{C}_{6} \mathrm{H}_{4}$ & 85 & $236-238$ & $2 \mathrm{~h}$ \\
9 & $4-\mathrm{OH} \mathrm{C}_{6} \mathrm{H}_{4}$ & 83 & $220-222$ & $2 \mathrm{i}$ \\
10 & $4-\mathrm{OCH}_{3} \mathrm{C}_{6} \mathrm{H}^{4}$ & 88 & $208-210$ & $2 \mathrm{j}$ \\
\hline
\end{tabular}

a: Yield of isolated products 


\section{RESULT AND DISCUSSION}

1-arylidene-2-(5-(4-methoxyphenyl)thiazol2-yl)hydrazine (2a-h, Table-1) were synthesized according to scheme 1 . Thiazole derivatives $2(a-h)$ were prepared by one pot two step condensation of aromatic aldehydes, thiosemicarbazide and phenacyl bromide. In first step aromatic aldehydes 1a-h $(1 \mathrm{mmol})$, thiosemicarbazide $(1 \mathrm{mmol})$ were reacted in ethanol at room temperature catalyzed by $10 \mathrm{~mol} \%$ sulphamic acid. It was resulted in thiosemicarbazone of corresponding aromatic aldehyde. Further same flask was charged with phenacyl bromide (1 mmol) and acidic buffer, stirred till completion of reaction. Completion of reaction was monitored by thin layer chromatography technique. After completion of reaction mixture was treated with cold water, separated solid was filtered, dried and recrystallized. The products were obtained in moderate to good yield. The structures of compounds 2(a-h) were confirmed by spectroscopic methods such as IR, ${ }^{1} \mathrm{HNMR},{ }^{13} \mathrm{C}$ NMR and mass spectroscopy. IR spectrum of 1-arylidene-2-(5-(4-methoxyphenyl)thiazol-2-yl) hydrazine 2(a-h) showed an absorption band around the $3000-3242 \mathrm{~cm}^{-1}$ regions resulting from the - $\mathrm{NH}$ - group. The absorption bands around 1570 and
$1487 \mathrm{~cm}^{-1}$ region resulting from $\mathrm{C}=\mathrm{N}$ (azomethine) and $\mathrm{C}=\mathrm{N}$ (thiazole) respectively. Fig. 1 showed IR spectra for compound 2d. In the ${ }^{1} \mathrm{HNMR}$ spectra, of compounds 2(a-h) showed a singlet proton at $12.00-12.50 \mathrm{ppm}$ for the $-\mathrm{NH}$ - and a singlet around at 7.90-8.45 ppm for the $-\mathrm{CH}-\mathrm{N}=$ proton the presence of a singlet proton at 7.15 for the thiazole. Peak near 3.70-3.80 ppm in spectra of all synthesized compounds is due to $-\mathrm{OCH}_{3}$ group of aromatic ring. Fig. 2 showed ${ }^{1} \mathrm{H}$ NMR spectra for compound $2 \mathrm{~d}$. The ${ }^{13} \mathrm{C}$ NMR studies indicate azomethine, aromatic and thiazole ring carbon in between $\delta 136-139$, 101-160 and around 167 respectively. Fig. 3 showed ${ }^{13} \mathrm{CNMR}$ spectra for compound $2 \mathrm{~d}$. The compounds were finally confirmed by mass analysis. Fig. 4 showed mass spectra for compound $2 \mathrm{~d}$.

From the above study it can be concluded that, the one pot two step protocol for the synthesis of 1-arylidene-2-(5-(4-methoxyphenyl)thiazol-2-yl) hydrazine 2(a-h) has been developed. It involves formation of thiosemicarbazone in first step and then cyclization with phenacyl bromide in second step. It has certain advantages such as facile, mild reaction condition, efficient, runs at room temperature, simple work-up and results in good yields.

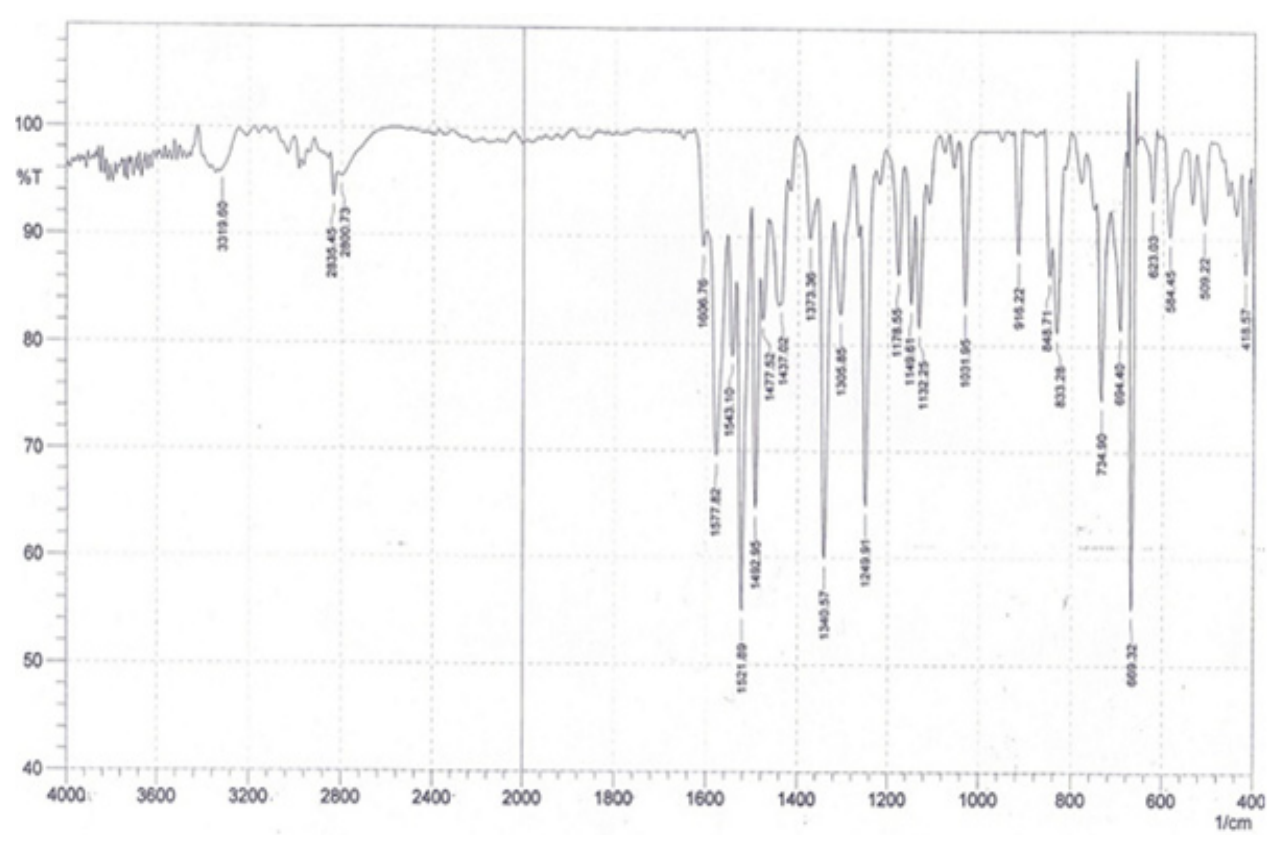

Fig. 1. IR Spectra of 4-(4-methoxyphenyl)-2-(2-(2-nitrobenzylidene)hydrazinyl)thiazole(2d) 


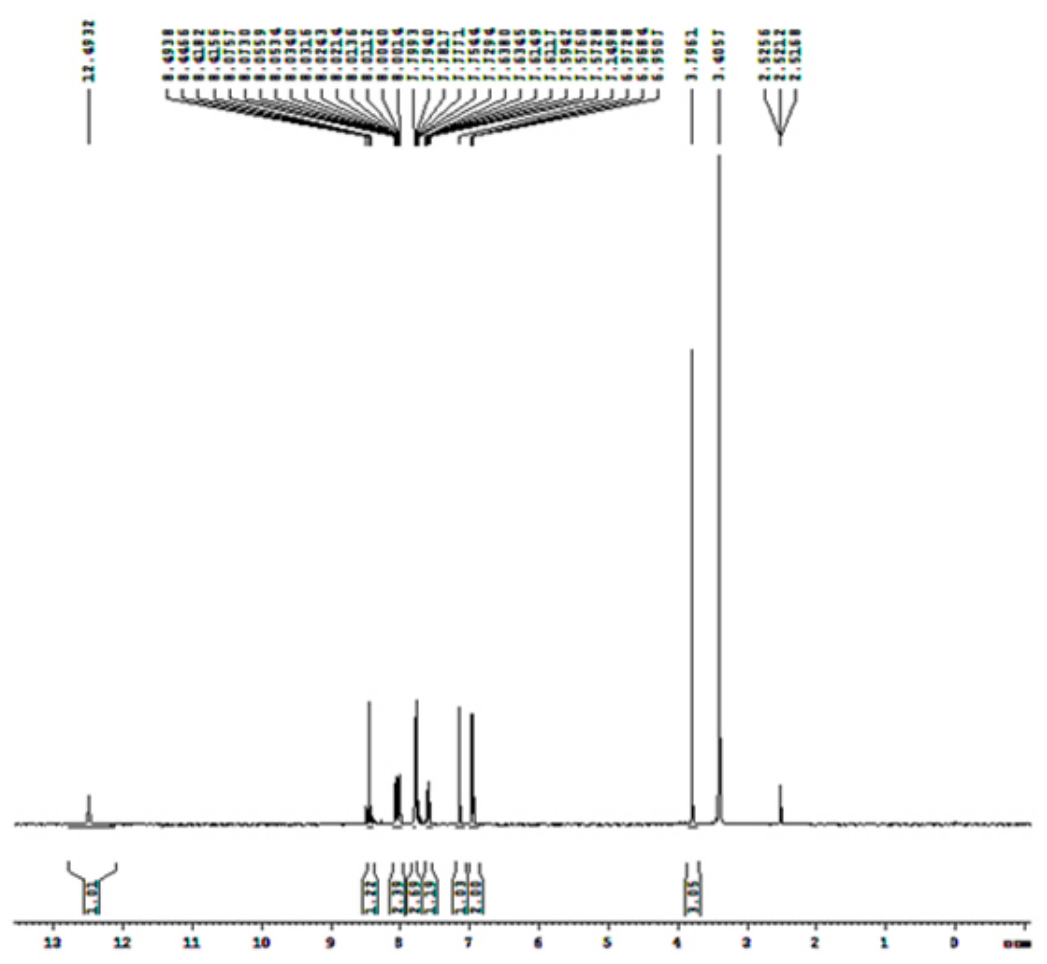

Fig. 2. ${ }^{1} \mathrm{H}-\mathrm{NMR}$ of 4-(4-methoxyphenyl)-2-(2-(2-nitrobenzylidene) hydrazinyl)thiazole(2d)

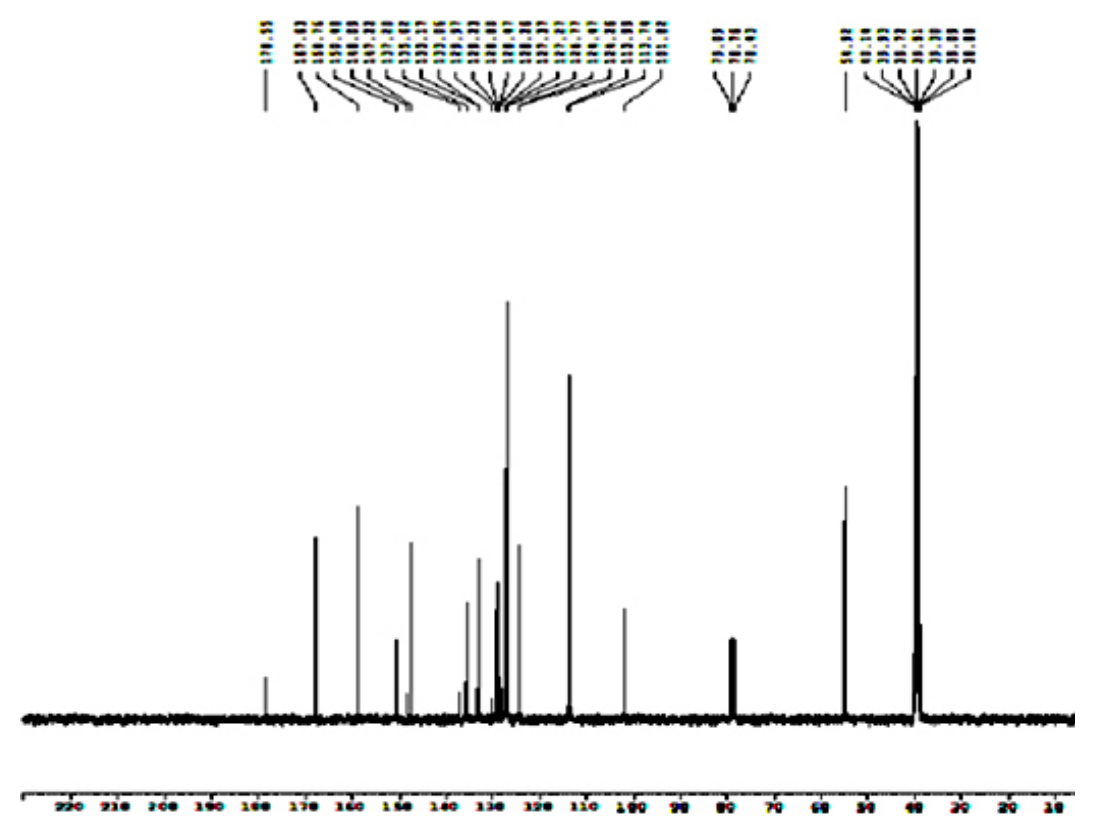

Fig. 3. ${ }^{13} \mathrm{C}$ NMR of 4-(4-methoxyphenyl)-2-(2-(2-nitrobenzylidene) hydrazinyl)thiazole(2d) 


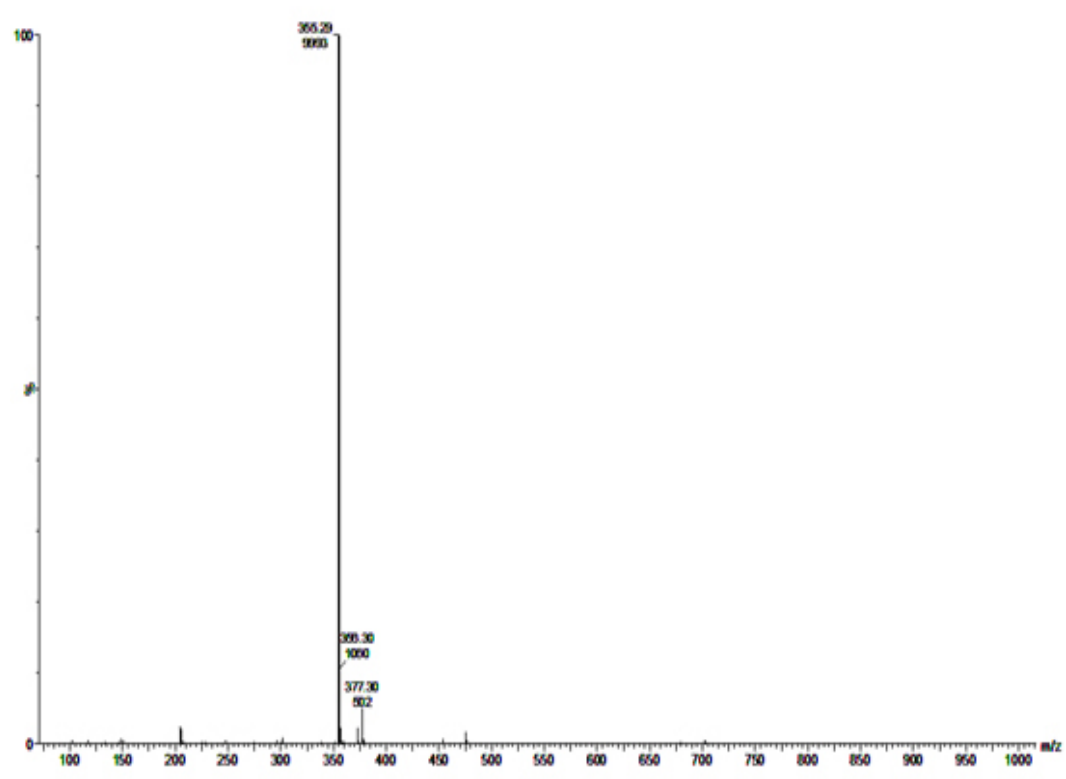

Fig. 4. Mass spectra of 4-(4-methoxyphenyl)-2-(2-(2nitrobenzylidene) hydrazinyl)thiazole(2d)

\section{ACKNOWLEDGEMENT}

Author is thankful to UGC (WRO), New
Delhi (Grant No. 47-1986/11 (WRO)) for financial assistant \& Director, SAIF, Panjab University, Chandigarh for providing spectral analysis facility.

\section{REFERENCES}

1. Cukurovali, A.; Yilmaz, I.; Gur, S.; Kazaz, C. Eur. J. Med. Chem., 2006, 41, 201-207.

2. Wilson, K. J.; Illig, C. R.; Subasinghe, N.; Hoffman, J. B.; Rudolph, N. J.; Soll R.; Molloy C. J.; Bone, R.; Green, D.; Randall, T.; Frank, M. Z.; Lewandowski F. A.; Zhou, Z.; Sharp, C.; Maguire, D.; Grasberger, B.; DesJarlais, R. L.; Spurlino, J. Bioorg. Med. Chem. Lett. 2001, 11, 915-918.

3. a) Berlin, K. D. ; Herd, M. D.; Proc. Okla. Acad. Sci., 1991, 71, 29-33. b) Helal, M. H. M.; Salem, M. A.; El-Gaby, M. S. A.; Aljahdali, M. Eur. J. Med. Chem., 2013, 65, 517-526. c) Sharma, P. K.; Sawnhney, S. N.; Gupta, A.; Singh, G. B.; Bani, S. Indian. J. Chem., 1998, 37, 376-381.

4. Pawar, C. D.; Sarkate, A. P.; Karnik, K. S. Bahekar, S. S.; Pansare D. N.; Shelke, R. N.; Jawale, C. S.; Shinde, D. B. Bioorg. Med. Chem. Lett., 2016, 26, 3525-3528.

5. John, L.; Kane, Jr.; Hirth, B. H.; Liang, B.; Gourlie, B. B.; Nahill, S.;Barsomian, G.; Bioorg. Med. Chem. Lett., 2003, 13, 4463-4466.
6. Naik, B.; Desai, K. R. Indian J Chem., 2006, 45, 267-271.

7. Venkatachalam, T. K.; Sudbeck, E. A.; Mao, C.; Uckun, F. M. Bioorg. Med. Chem. Lett., 2001, 11, 523-528.

8. Wilkes, M. C.; Lavrik, P. B.; Greenplate, J. Journal of Agricultural Food Chemistry., 1991, 39, 1652-1657.

9. Warhurst, D. C.; Agadu, I. S.; Nolder, D.; Rossignol, J. F.; J. of Antimicrobial Chemotherapy., 2002, 49, 103-111.

10. Fahmy, H. T.; Bekhit, A. A. Pharmazie., 2002, 57, 800-803.

11. Lu, Y.; Li, C. M.; Wang, Z.; Ross, C. R.; Chen, J.; Dalton, J. T.; Li, W.; Miller, D. D.; J. Med. Chem., 2009, 52, 1701-1711.

12. Carbone, A.; Pennati, M.; Parrino, B.; Lopergolo, A.; Barraja, P.; Montalbno, A.; Spanò, V.; Sbarra, S.; Doldi, V.; de Cesare, M. J. Med. Chem., 2013, 56, 7060-7072.

13. Steinbaugh, B. A.; Batley, B. L.; Painchaud, C. A.; Rapundalo, S. T.; Michniewicz, B. M.; Olson, 
S. C. J. J. Med. Chem., 1992, 35, 2562-2572.

14. a) Zaharia, V.; Ignat, A.; Palibroda, N.; Ngameni, B.; Kuete, V.; Fokunang, C. N.; Moungang, M. L.; Ngadjui, B.T. Eur. J. Med. Chem., 2010, 45, 5080-5085. b) Mohammad, S. A.; Lijun, L.; Yong-Eok, L.; Dong-Ung, L. Chem. Pharm. Bull., 2011, 59, 568- 573.

15. Yeong, J. J.; Wen, W. L.; Yuh, K. S.; Ju, T. L.; Ming, H. H.; Ching, F. Y. Tetrahedron., 2003, 59, 4979-4992.
16. Saikia, L.; Roudragoud, P.; Thakur, A. J. Bioorganic \& Medicinal Chemistry Letters, 2016, 26, 992-998.

17. Tokmajyan, G.; Karapetyan L. Heterocycl. Commun., 2014, 20, 77-79.

18. Hernandez, A. G.; Grooms, G. M.; El-Alfy, A. T.; Stec J. Synthesis., 2017, 49, 2163-2176.

19. Tryambake, P.T. Der Pharma Chemica., 2017, 9, 144-152. 